\title{
EFFECTS OF QUANTITATIVE ELECTROENCEPHALOGRAM NORMALIZATION USING 4-CHANNEL LIVE Z-SCORE TRAINING NEUROFEEDBACK FOR CHILDREN WITH LEARNING DISABILITIES: PRELIMINARY DATA
}

\author{
Rubén Pérez-Elvira ${ }^{1}$, Javier Oltra-Cucarella² \\ and José Antonio Carrobles ${ }^{3}$ \\ ${ }^{1}$ NEPSA Neurological Rehabilitation, Salamanca; ${ }^{2}$ Miguel Hernández \\ University, Elche; ${ }^{3}$ Autonomus University of Madrid (Spain)
}

\begin{abstract}
Children with learning disabilities (LD) can have difficulties in reading at word level, text comprehension, writing or arithmetic. Several studies have shown the efficacy of neurofeedback (NF) in improving learning skills through brainwave operant conditioning in children with LD. The aim of this work was to show the efficacy of live z-score NF training (LZT) for quantitative electroencephalogram (QEEG) normalization in school children with LD. Twenty-eight children aged 1015 years with LD participated. Ten 30-min sessions of QEEG-guided LZT using patient's highly preferred feedback were applied. After 10 sessions of QEEGguided LZT, participants showed statistically significant improvements in QEEG normalization and a statistically significant small to medium improvement in the Cognitive and Emotional Checklist. The results suggest that LZT-NF produces a tendency towards normalization of brain waves in children with LD, and might be advised as a therapeutic alternative or coadjuvant along with cognitive interventions.

KEY WORDS: Neurofeedback, z-score training, QEEG normalization, learning disabilities.
\end{abstract}

\section{Resumen}

Los niños con trastornos de aprendizaje (TA) pueden manifestar dificultades en la lectura a nivel de palabra, comprensión de textos, escritura o matemáticas. Algunos estudios han mostrado la eficacia de la neurorretroalimentación (NR) en la mejora de las habilidades de aprendizaje a través del condicionamiento operante de las ondas cerebrales en niños con TA. El objetivo de este trabajo fue mostrar datos preliminares de la eficacia de la neurorretroalimentación de puntuaciones $z$ en tiempo real (LZT) para la normalización del electroencefalograma cuantitativo (QEEG) en escolares con TA. Participaron 28 niños de entre 10-15 años de edad con TA. Se aplicaron diez sesiones de 30 minutos de LZT guiado por QEEG utilizando retroalimentación de alta preferencia de los sujetos. Tras 10 sesiones de LZT guiado por QEEG, los participantes

Correspondence: Javier Oltra-Cucarella, Dpt. of Health Psychology, Miguel Hernández University, Elche (Spain). E-mail address: joltra@umh.es 
mostraron mejoras estadísticamente significativas en la normalización del QEEG y una mejora de pequeña a media estadísticamente significativa en la "Lista de comprobación cognitiva y emocional". Los resultados sugieren que LZT-NR produce una tendencia hacia la normalización de las ondas cerebrales en niños con TA, y podría suponer una alternativa terapéutica o coadyuvante junto con la intervención cognitiva.

PALABRAS CLAVE: Neurorretroalimentación, entrenamiento en puntuaciones z, normalización del QEEG, trastornos de aprendizaje.

\section{Introduction}

The identification of children learning disabilities (LD) in the school context is one of the main goals for school psychologists (Orlando \& Rivera, 2004). LD may be related to medical, psychological or pedagogical problems, among others (Orozco Cazco et al., 2016), and place the student in what has been called special educational needs (SEN) (Lindsay, 2007). The term SEN is used to refer to students who need specific support or attention during either a part or all of their schooling (Orozco Cazco et al., 2016). Students with LD have difficulties in cognitive processes such as reading at word level (dyslexia), text comprehension (reading comprehension), writing (dysgraphia) or arithmetic (dyscalculia) (Fletcher \& Grigorenko, 2017).

In addition to psychological or academic interventions for LD (Fletcher \& Grigorenko, 2017), several studies have been conducted lately on the efficacy of neurofeedback (NF) for children with LD. NF is a psychophysiological technique that focus on the modification of the brain activity reflected in the electroencephalogram (EEG). Via an EEG amplifier, the patient's activity is recorded, which is then broken down into frequency bands, and the EEG pattern is modified by operant conditioning, providing a reinforcement when the patient achieves the desired EEG pattern (Carrobles, 2016).

Several studies have shown the efficacy of NF for children with LD. Orlando and Rivera (2004) applied NF to children with reading disabilities and found an improvement in psychometric scores after the intervention. In a controlled study with children with dyslexia, Breteler et al. (2010) applied NF and school support measures to the experimental group as opposed to the control group, which only received school support, and found that the experimental group improved significantly in spelling scores, with no differences between groups in reading tasks. Jacobs (2006) showed improvements in reading skills after applying NF in two adolescents with pluripathology. Fenger (1998) found improvements in academic performance tests in 38 children and adolescents aged 8-18 years after 46 sessions of NF. Hanselmayr et al. (2005) found an improvement in mental rotation tasks, a discovery replicated by Zoefel et al. (2011) in a training study of the upper alpha band in posterior locations. Koberda et al. (2012) used 19-channel live z-score NF (LZT-NF) training on a subject with academic performance problems, achieving an increase in scores on a global cognitive performance scale after 15 sessions. 
LZT-NF is an NF approach based on EEG quantification (QEEG) and the concept of standard deviation (Collura, 2014). Using LZT, the raw QEEG scores are to standardized scores (i.e., z-scores) using the distribution of the waves in a reference population, and can thus be incorporated as an element in a contingency system (Collura, 2014; Collura et al., 2010). It is a live mix of QEEG and NF. The z-scores from the QEEG are incorporated as variables so that the patient can get the reinforcement each time the z-score lies between the upper and lower ranges established in the intervention.

Because the abnormalities found in the QEEG are stable in the absence of intervention (Hughes \& John, 1999), the QEEG provides an objective measure of the effects of intervention on brain functioning (Arns et al., 2012; Walker, 2011, 2010; N. Wigton \& Krigbaum, 2015; N. L. Wigton \& Krigbaum, 2015). Collura et al. $(2008 ; 2010)$ add that the use of 4-channel LZT protocols with F3, F4, P3 and P4 locations ensures sufficient coverage for an overall normalization of QEEG, an issue that has also been observed by other authors (Groeneveld et al., 2019).

Wigton and Krigbaum (Krigbaum \& Wigton, 2015a; Wigton \& Krigbaum, 2015; Wigton \& Krigbaum, 2015) reported that there are few studies that include pre- and post-intervention QEEG data, provide a methodology for assessing normalization, and highlighted the need to replicate their methodology to give greater integrity to their results. Their methodology for normalization analysis allows monitoring both the treatment progression and QEEG changes both during and at the end of treatment. In a retrospective study (Krigbaum \& Wigton, 2015a) they selected 10 subjects between the ages of 10 and 56 with different diagnoses who had received a maximum of 15 LZT sessions. They determined the locations to train based on the dysregulation found in the QEEG, considering the region of interest (Rol) those where a z-score $> \pm 1$ was found. They calculated the mean of the positive and negative $Z$ scores separately in each Rol and used the resulting score as a follow-up value, and found that $90 \%$ of the Rol showed a trend towards the mean (i.e., normalization) after the intervention. Using the mean of the absolute scores in each Rol, Wigton and Krigbaum (2015) generated a single deviation score from the mean for each participant. After applying about 10 LZT sessions on average, they again evaluated the distance to the mean, obtaining a value closer to the mean compared to the pre-treatment phase.

Following the methodology of Krigbaum and Wigton (2015a), the objective of this work is to obtain preliminary data of the normalization effect of QEEG using an LZT protocol in a sample of children with LD. To this end, this work was specifically designed to analyze a) whether the LZT protocol produces a change in the percentage of QEEG z-scores within the normal range, and b) to quantify the degree of normalization in the waves used during the intervention. Following the results reported in previous works showing the efficacy of NF as an intervention for children with LD, we expected to find significant improvements in clinical outcomes. Additionally, and to expand the data reported in previous works using NF for children with LD, we aimed to quantify the changes that occurred on clinical outcomes after a LZT-NF intervention. 


\section{Method}

\section{Participants}

Data for 28 children (25 boys) aged 12.07 years on average $(S D=1.58$, range: 10-15) were analyzed after excluding records with missing data or diagnoses unrelated to learning disabilities (LD). Table 1 shows the specific LD profile associated with each individual participant along with baseline descriptive data. Most of the participants had reading disabilities $(n=24)$, followed by problems with arithmetic $(n=9)$ and writing disabilities $(n=7)$. Ten out of 28 had two or more LD.

The inclusion criteria were: 1) being diagnosed with LD following the Government classification of students with LD (Instrucción de 24/08/2017de la Consejeria de Educación de la Junta de Castilla y León, Spain). Per protocol, children are diagnoses with LD by consensus among school psychologists and neuropediatricians; 2) being aged between 10 and 15 years; 3) having a QEEG pattern with multiple abnormal findings (i.e., more than one abnormal wave, in more than one location or region); 4) having completed 10 LZT sessions in F3, F4, P3 and P4 locations; 5) Having an average intelligence quotient estimate according to the Wechsler Intelligence Scale for Children - $4^{\text {th }}$ Ed (Kaufman et al., 2006).

Table 1

Learning disabilities profile for the sample

\begin{tabular}{|c|c|c|c|c|c|c|c|}
\hline Participant & Sex & Age & Learning disability & Participant & Sex & Age & Learning disability \\
\hline 1 & B & 10 & R & 15 & B & 14 & R \\
\hline 2 & B & 11 & R, A & 16 & B & 13 & R \\
\hline 3 & B & 10 & R & 17 & B & 14 & R \\
\hline 4 & B & 12 & R & 18 & B & 13 & R \\
\hline 5 & B & 12 & R & 19 & B & 11 & R \\
\hline 6 & B & 13 & R, W & 20 & B & 11 & R, A \\
\hline 7 & B & 11 & W, A & 21 & B & 12 & W \\
\hline 8 & B & 13 & A & 22 & B & 10 & R, W \\
\hline 9 & B & 15 & R, W, A & 23 & G & 14 & R \\
\hline 10 & B & 10 & R & 24 & G & 13 & R, A \\
\hline 11 & B & 11 & A & 25 & B & 10 & R \\
\hline 12 & G & 13 & R & 26 & B & 11 & R, W \\
\hline 13 & B & 12 & R, W, A & 27 & B & 12 & R \\
\hline 14 & B & 14 & R & 28 & B & 12 & R, A \\
\hline
\end{tabular}

Note: $\mathrm{B}=$ boy; $\mathrm{G}=$ girl; $\mathrm{R}=$ reading; $\mathrm{W}=$ writing; $\mathrm{A}=$ arithmetic.

\section{Instruments}

a) Discovery 20 channel device (BrainMaster Technologies, 2012). It is a 20channel neurofeedback (EEG biofeedback) device. It provides 24-bit conversion with an internal sampling rate of 1024 samples/second and 256 
samples/second data rate to the computer, providing 20 high-resolution and aliasing-free signals.

b) Electro-Cap (EEG Cap.) (Electro-Cap Internacional, 1982). it is an EEG electrode application technique. They are made of an elastic spandex-type fabric with recessed, pure tin electrodes attached to the fabric. The electrodes on the standard caps are positioned to the International 10-20 method of electrode placement. To record de EEG the electrodes reservoir need to be filled with Electro-Gel (Electro-Cap Internacional, 1982), a saline gel that favors conductivity. The medium Electro-Cap will fit approximately $65 \%$ of all patients over the age of 5 .

c) BrainAvatar 4.6.4 software (BrainMaster Technologies, 2013). This software was used to record the EEG.

d) NeuroGuide v. 2.9.1 (Applied Neuroscience, 2018). This software was used for EEG computation and analysis. This software includes a normative database of QEEG from 2 months to 82 years of age.

e) Cognitive Emotional Checklist (CEC; Soutar, 2018). The CEC is an inventory to collect information about emotional, cognitive, and behavioral symptoms. The CEC was developed for the use of NF practitioners to assist them in better understanding the cognitive and emotional status of their clients and monitor follow-up changes in that status (Soutar, 2018). The CEC includes 49 items that are answered by parents on a 4-point Likert-type scale, ranging from 0 (no symptom) to 3 (present and severe symptom). Scores can range between 0-147, with higher scores indicating a greater severity of the symptomatology. In order to verify the impact of the intervention on educational difficulties, the 10 CEC items related to learning, mathematics, reading and writing (CECLearning) were analysed as a separate outcome. As in the rest of the CEC, the sum of scores for these items ranges from 0 to 30, with higher scores indicating greater frequency and severity of the problems. The main outcomes were the CEC Total score and the CEC-Learning score.

\section{Procedure}

The participants' parents signed an informed consent for the application of the treatment and for the subsequent anonymized use of data for research purposes. The intervention was delivered in NEPSA Neurological Rehabilitation, a neurological rehabilitation clinic authorised by Government of Castilla y León (Spain). The Health Department gave approval for psychophysiological interventions of this type within the context of psychological treatments.

An EEG was obtained from each participant before the start of the NF sessions and after 10 LZT sessions. For the collection of the EEGs each patient was fitted with an electroencephalography cap, Electro-Cap (Electro-Cap Internacional, 1982), with 19 channels located according to International System 10-20 (Fp1, Fp2, F7, F3, Fz, F4, F8, T3, C3, Cz, C4, T4, T5, P3, Pz, P4, T6, O1, and O2) and using a Linked Ears assembly. For 3-5 minutes, EEG signals from all 19 channels were simultaneously obtained and collected using a Discovery20 amplifier from BrainMaster Technologies (2012). Impedances of less than 5KOhms were 
maintained. EEG records were recorded in closed eye condition using Brain Avatar 4.6.4 software (BrainMaster Technologies, 2013).

The EEG records were imported into NeuroGuide v. 2.9.1 (Applied Neuroscience, 2018) software for computation and analysis, where the artefacts were visually reviewed and removed. EEG records were processed using Linked Ears assembly and compared to NeuroGuide's normative database, from which zscore values were obtained. Seven frequency bands were selected (delta, theta, alpha, beta-1, beta-2, beta-3 and hibeta). Beta was excluded because its activity was already included in the breakdown (beta-1, beta- 2 and beta-3). Similarly, relative power was excluded as it was a calculation of the absolute power distribution of the entire spectrogram. In this way, redundant data was avoided (Stoller, 2011), which allowed each wave to be treated as a variable independent of the rest of the variables. Afterwards, parents completed the CEC.

INTERVENTION: LIVE Z-SCORES TRAINING NEUROFEEDBACK

A QEEG-guided LZT protocol was used. Because all patients had a QEEG pattern with multiple and diffuse findings, F3, F4, P3 and P4 locations were selected in an LZT PZOKUL protocol because of their capacity for global normalization (Collura, 2008). A Linked Ears assembly was used with ground in Cz. The PZOKUL protocol has a threshold for the percentage of z-scores that must fall within the stablished deviation range, and the threshold is self-adjusted. This selfadjustment is based on the percentage of z-scores that fall within the set deviation range, with an upper threshold (positive $z$-scores) and a lower threshold (negative $z$-scores), and the percentage of reinforcement that the patient is achieving. In order to avoid that the movements from inside to outside the normal range could not be identified, due to a wide range for the z-score window (Collura, 2014; Collura et al., 2010), $z$-scores equal to or more extreme than \pm 1.5 were categorized as out of range. Considering the above, 28 values were obtained for each subject in Z-scores, four (F3, F4, P3 and P4) for each wave.

Patients were given 30-minute sessions twice a week without interruption until 10 LZT sessions were completed, at which time a follow-up QEEG was repeated. Participants were allowed to choose both the form (i.e., visual/auditory) and type of feedback in each session, based on the results of previous studies indicating that the more relevant the enhancer is to the subject the greater the learning effect is produced (Fisher et al., 1992; Mangum et al., 2012; Piazza et al., 1996). In all cases, the selected enhancers were movies chosen by each participant.

During the sessions, all z-scores for each of the 7 bands selected for each channel were computed moment by moment. Likewise, the percentage of those scores within the specified range $( \pm 1.5 \mathrm{SD})$ was calculated moment by moment, and the participants received reinforcement each time the percentage of z-scores within that range was equal to or greater than the percentage requested by the software as criterion to achieve reinforcement. This was automatically calculated to guarantee a 50\% reinforcement (Pérez-Elvira, et al., 2019).

In order to produce feedback, a dimmer was overlapped on the screen where the films were projected. The dimmer became clear when the subject met the LZT 
protocol criteria for receiving feedback and became opaque, preventing the patient from viewing the film, when it moved away from the criteria set out in the protocol.

\section{Data analyses}

To analyze change in the QEEG metrics, each of the 28 waves for each participant was dichotomized as 1 when their z-score absolute value was equal or higher than 1.5 (i.e., out of normal range), otherwise as 0 . The dependent variable in this study was pre-post intervention change in the percentage of QEEG waves out of normal range. The difference in the likelihood of change after NF was analyzed with a binary logistic model using a Generalized Estimating Equations (GEE) with an independent correlation structure and robust standard errors. The GEE is a statistical approach that accounts for the correlation between measurements in clustered data (i.e., variables grouped by a cluster identification variable). In the present study, QEEG z-scores were clustered by participants. Thus, clusters are independent of one another but the within cluster observations are assumed to be correlated. Both pre and post raw z-scores for the 28 waves were averaged within participants, and mean z-score PRE/POST change and mean CEC PRE/POST change were analyzed with paired t-tests. Effect sizes were calculated using the variance-weighted single-group pretest-posttest effect size (Morris \& DeShon, 2002). Although this effect estimate is not analogous to the independent samples Cohen's d statistic (Cohen, 1992), it is interpreted using Cohen's guidelines, with $0.20,0.50$ and 0.80 indicating a small, medium, and large effect respectively. Statistical analyses were run with IBM SPSS Statistics v. 26.0. Alpha level was set at $5 \%$.

\section{Results}

Tables 2 shows the QEEG and clinical profile data at baseline, and the mean age of the participants, both for those who had $1 \mathrm{LD}$ or more than $1 \mathrm{LD}$. Mean pre and post absolute $z$-scores were $2.05(S D=0.34)$ and $1.41(S D=0.43)$ respectively, with this difference being large and statistically significant $\left(t_{27}=8.57, p<.001, d=-\right.$ 1.86). Overall, $42.5 \%$ of the waves $(n=333)$ were out of the normal range at pretest, compared to $25.1 \%(n=197)$ at post-test. Figure 1 shows z-scores for each wave by channel both a pre- and post-intervention. The GEE (Figure 2) showed that waves out of the normal range at pre-test were more likely to change (i.e., to move towards the normal range at post-test) than did the waves within the normal range at pre-test $(b=2.77, \mathrm{SE}=0.44, \mathrm{OR}=15.95,95 \% \mathrm{Cl}: 6.73,37.80)$. Results were unchanged when age and the number of LDs were added as covariates in the model, with neither age $(b=0.08, S E=0.13, \mathrm{OR}=1.08,95 \% \mathrm{Cl}: 0.83,1.39)$ nor the number of $\mathrm{LDs}(\mathrm{b}=0.07, \mathrm{SE}=0.36, \mathrm{OR}=1.08,95 \% \mathrm{Cl}: 0.54,2.17)$ showing a statistically significant association with pre-post change.

According to the parent reports, mean CEC-Total scores decreased from $49.96(S D=8.24)$ at pre-test to $33.43(S D=7.29)$ at post-test, with this difference being small to medium and statistically significant $\left(t_{27}=14.37, p<.001, d=-0.46\right)$. 
Likewise, mean CEC-Learning scores decreased from $18.29(S D=3.18)$ at pre-test to $11.39(S D=2.69)$ at post-test, with this difference being small to medium and statistically significant $\left(t_{27}=14.26, p<.001, d=-0.43\right)$. Figures 3 and 4 show prepost change in CEC-Total scores and CEC-Learning scores respectively.

\section{Table 2}

Baseline demographics, QEEG and LD data

\begin{tabular}{|l|c|c|c|c|c|c|}
\hline \multirow{2}{*}{\multicolumn{1}{|c|}{ Variables }} & \multicolumn{2}{c|}{$\begin{array}{c}\text { Number of } \mathrm{LD}=1 \\
(n=18)\end{array}$} & $\begin{array}{c}\text { Number of } \mathrm{LD}=2+ \\
(n=10)\end{array}$ & \multirow{2}{*}{$z$} & \multirow{2}{*}{$p$} \\
\cline { 2 - 6 } & $M$ & $S D$ & $M$ & $S D$ & & \\
\hline Age & 12.04 & 1.37 & 12.03 & 1.50 & -0.49 & .625 \\
\hline Z-scores pre-test $(n=504)$ & 1.23 & 0.86 & 1.01 & 0.81 & -1.61 & .108 \\
\hline Z-scores post-test $(n=280)$ & 0.94 & 0.66 & 0.84 & 0.56 & -0.46 & .649 \\
\hline CEC Total pre-test & 48.76 & 7.57 & 51.59 & 8.51 & -1.95 & .052 \\
\hline CEC Total post-test & 32.74 & 6.98 & 34.36 & 7.33 & -0.29 & .773 \\
\hline CEC Learning pre-test & 18.00 & 3.11 & 22.00 & 1.41 & -0.29 & .773 \\
\hline CEC Learning post-test & 11.08 & 2.53 & 15.50 & 0.71 & -1.60 & .121 \\
\hline
\end{tabular}

Note: $\mathrm{LD}=$ learning disabilities; $\mathrm{CEC}=$ Cognitive and Emotional Checklist.

Figure 1

Pre-post z-scores maps

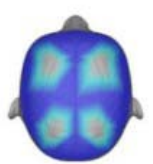

Delta

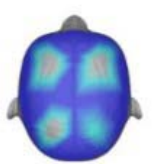

Theta

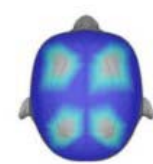

Alpha

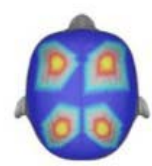

Beta1

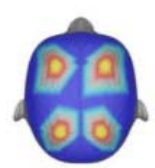

Beta2

\section{Z-Score}

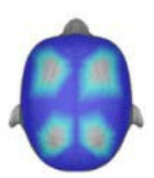

Delta

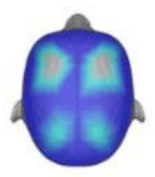

Theta

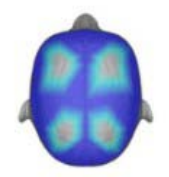

Alpha

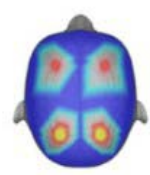

Beta1

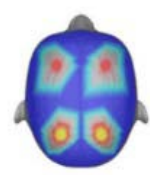

Beta2

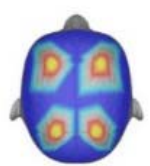

Beta3

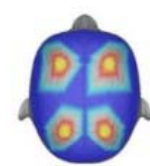

HiBeta

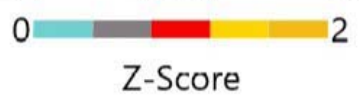

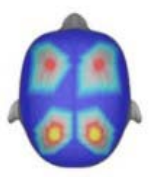

Beta3

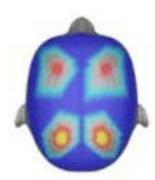

HiBeta

\section{Z-Score}

Note: Z-scores maps for each band and location pre (top) and post (bottom) neurofeedback intervention (maps from a modification of those produced by New Mind Maps software). 
Figure 2

Pre-post change in z-scores out of normal range

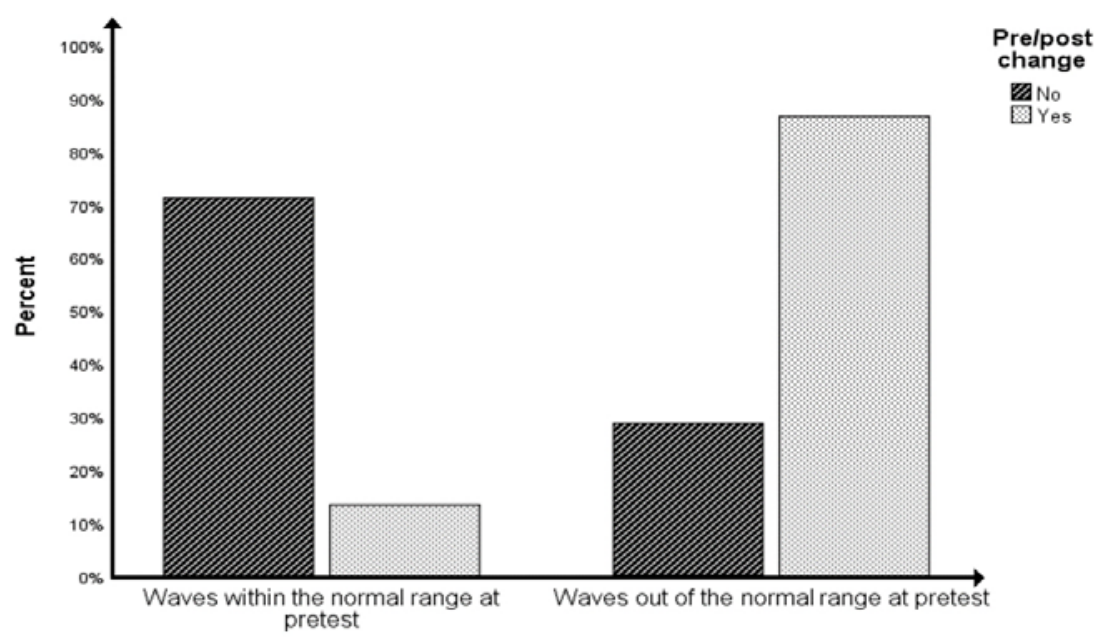

Figure 3

Pre-post change in Cognitive and Emotional Checklist Total scores

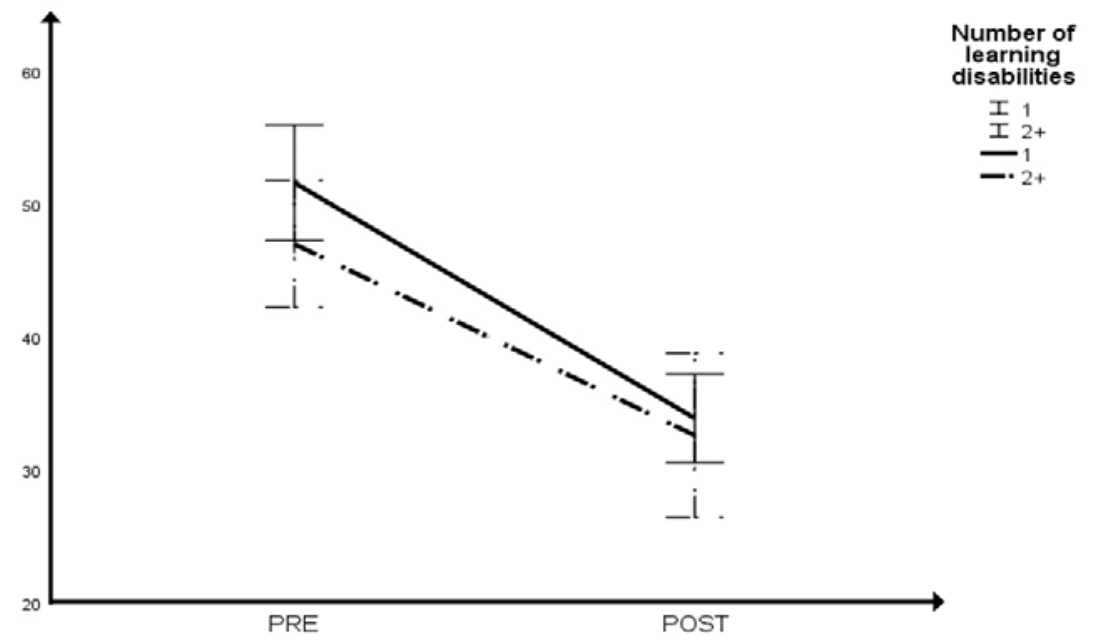




\section{Figure 4}

Pre-post change in Cognitive and Emotional Checklist - Learning scores

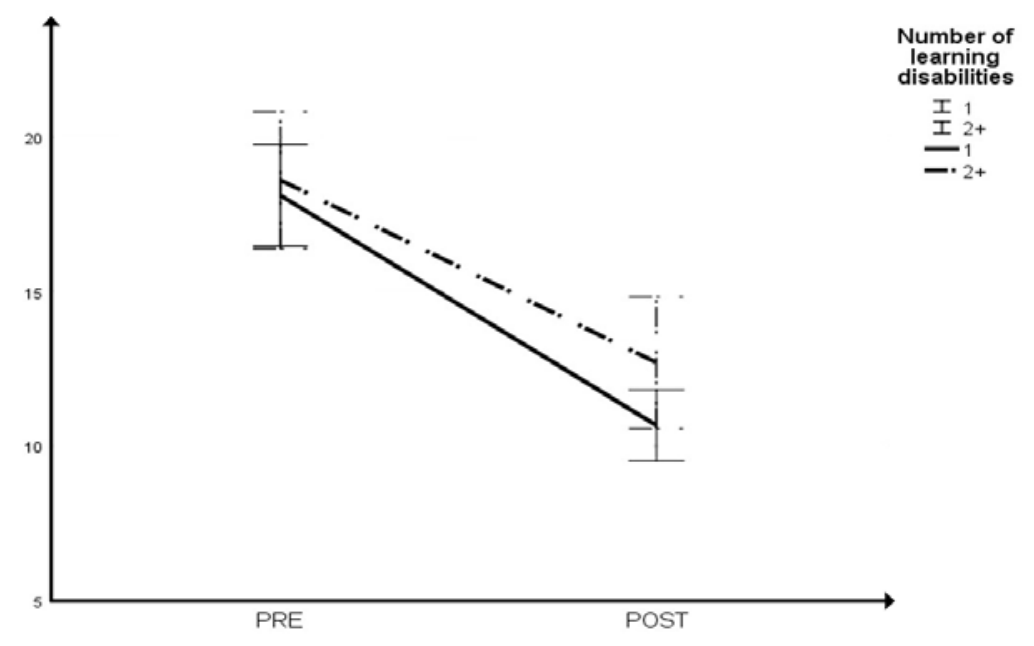

\section{Discussion}

The present work aimed to study the efficacy of a 4-channel LZT-NF intervention for the normalization of the QEEG pattern in patients with LD. To do so, a variation of the method developed by Wigton and Krigbaum (2015a) was used with F3, F4, P3 and P4 locations, given the ability of this arrangement to produce a global normalization trend (Collura, 2008).

Our results showed that the LZT protocol was effective in producing the trend towards QEEG normalization after ten 30-minute sessions, with an association between this QEEG normalization and an improvement in the clinical variables measured through the parents' perception of each participant's specific problems.

While some authors have suggested that great caution should be taken when seeking QEEG normalization (Kropotov, 2009, 2016), it does not, in any case, appear to pose any significant risks (Collura et al., 2010). We found that the odds of moving toward the standard value was greater than the odds of moving outside the standard values after treatment, which is in line with previous studies (PérezElvira et al., 2018; Pérez-Elvira, et al., 2019) reporting that there is little likelihood for z-scores to move out of the normal range due to the LZT intervention.

Some authors (Azizi et al., 2018) have concluded that NF is not effective for LD. They compared NF with cognitive rehabilitation for LD, but their study has some methodological limitations. For instance, they studied the effect of NF on sustained attention in LD, and used the Theta-to-Beta protocol that was developed in the first place for the treatment of epilepsy, and was later applied as a one-sizefits-all procedure for other conditions including Attention Deficit and Hyperactivity Disorder. To support the use of this methodology, these authors argued that some children with LD have also sustained attention issues. We believe that it cannot be 
concluded that NF is not effective for LD, but rather that NF applied using the Theta-to-Beta protocol might not be effective to improve impairments in sustained attention. These conclusions, however, are not in line with previous works showing contradictory findings (Kaiser \& Othmer, 2000; Medici, 2018; Ochi et al., 2017; Rastegar et al., 2016; Wang \& Hsieh, 2013).

Our results are similar to those found by several authors (Fenger, 1998; Jacobs, 2006; Orlando \& Rivera, 2004), who applied QEEG-NF protocols to children with $L D$, obtaining significant improvements in those who received the treatment as compared with the control group, along with a lower rate of early treatment dropout (N. L. Wigton, 2013). Compared to the present work, however, some of these studies (Fenger, 1998; Jacobs, 2006) reported positive results just after 40 or more sessions. One possible explanation for this is that they did not use QEEG-NF but symptom-guided NF. As several authors have stated, the average number of sessions needed to produce significant changes with traditional methods, including amplitude QEEG-NF, is around 40 (Krigbaum \& Wigton, 2014, 2015b; Thatcher, 2013; Wigton, 2013; Wigton \& Krigbaum, 2015).

In the present study, clinically significant results were found in only 10 sessions, which is a particular strength compared to other NF studies for intervention in children with LD. Our results are comparable to those reported by Wigton and Krigbaum (2015), Krigbaum and Wigton (2015a) and Groeneveld et al. (2019), who found not only improvement of symptoms but also normalization of QEEG within 30 sessions, or less, using LZT. The faster achievement of objectives under LZT could be due to the multidimensional character of the training it produces. As noted above, rather than targeting amplitudes, LZT focuses on the variables approaching the expected normal values according to the normative reference population (Groeneveld et al., 2019; Krigbaum \& Wigton, 2014). The focus with LZT is not to increase or decrease the amplitude of the neurophysiological variables, but to move the z-scores towards the mean and normalize the QEEG (Krigbaum \& Wigton, 2014, 2015b; Wigton \& Krigbaum, 2015). The $z$-scores that must be within the marked range are not pre-specified, only the percentage of those that must be within the range. In this way, this requested percentage will sometimes be composed of some scores and other times by others, thus giving the brain the opportunity to establish its own route towards self-regulation (Collura, 2008, 2014, 2016; Collura et al., 2010). When the brain achieves reinforcement by complying with the established percentage, it can compute the cost-benefit of achieving that percentage and thus learn the most relevant behavior to achieve the objective (Collura, 2014).

This study has some limitations that must be highlighted. On the one hand, it is a retrospective study, which limits the availability to include relevant variables and affects the sample size. Furthermore, in order to control the improvement of clinical variables, we used the CEC (Soutar, 2018), a test that is routinely applied in our clinic. Although it is very useful for tracking symptoms and reflecting the parents' view of the child's condition in different fields, the CEC Learning items do not allow any determination of the specific difficulties/improvements following the intervention. Further psychometric validation is required for research settings. 
Another limitation is the lack of a control group, which would help to monitor any possible pre-post changes in brain waves without intervention. However, it has been reported that the QEEG pattern associated with a pathology remains unchanged in the absence of treatment (Hughes \& John, 1999). Therefore, pre-post changes in brain waves should not be expected to occur only by chance. Future works will analyze whether LZT provides additional benefits over other NF interventions. Despite this, a strength of the present work is the inclusion of single-group pre-post effect size using a meta-analytic approach, which makes our data useful to be included in future meta-analyses on the efficacy of NF interventions for children with LD.

In summary, our study shows preliminary positive data on the efficacy of LZT for the normalization of EEG in children and adolescents with LD after 10 sessions of QEEG-NF, and its associated improvements in scholar and behavioral problems reported by their parents.

\section{References}

Applied Neuroscience. (2018). NeuroGuide (Version 2.9.1) [Computer software]. https://appliedneuroscience.com/product/neuroguide/

Arns, M., Drinkenburg, W., \& Leon Kenemans, J. (2012). The effects of QEEG-informed Neurofeedback in ADHD: An open-label pilot study. Applied Psychophysiology and Biofeedback, 37(3), 171-180. doi: 10.1007/s10484-012-9191-4

Azizi, A., Drikvand, F. M., \& Sepahvandi, M. A. (2018). Comparison of the effect of cognitive rehabilitation and neurofeedback on sustained attention among elementary school students with specific learning disorder: A preliminary randomized controlled clinical trial. Applied Psychophysiology and Biofeedback, 43(4), 301-307. doi: 10.1007/s10484-018-9410-8

BrainMaster Technologies (2012). Discovery-20 [Apparatus]. https://brainmaster.com/ product/discovery-20-series/

BrainMaster Technologies (2013). BrainAvatar (Version 4.6.4) [Computer software]. https://brainmaster.com/product/brainavatar-4-0-for-atlantis/

Breteler, M. H. M., Arns, M., Peters, S., Giepmans, I., \& Verhoeven, L. (2010). Improvements in spelling after QEEG-based neurofeedback in dyslexia: A randomized controlled treatment study. Applied Psychophysiology and Biofeedback, 35(1), 5-11. doi: 10.1007/s10484-009-9105-2

Carrobles, J. A. (2016). Bio/neurofeedback. Clínica y Salud, 27(3), 125-131. doi: 10.1016/j.clysa.2016.09.003

Cohen, J. (1992). A power primer. Psychological Bulletin, 112(1), 155-159. doi: 10.1037/0033-2909.112.1.155

Collura, T. (2008, April). Whole-head normalization using live z-scores for connectivity training. Neuroconnections. http://www.brainm.com/software/pubs/tfc/collura\%20nc $\% 20$ z\%201.pdf.

Collura, T. (2014). Technical foundations of neurofeedback. New York, NY: Routledge, Taylor \& Francis Group.

Collura, T. (2016). Live z-score Neurofeedback. Biofeedback, 44(4), 212-217. doi: 10.5298/1081-5937-44.4.01

Collura, T., Guan, J., Tarrant, J., Bailey, J., \& Starr, F. (2010). EEG biofeedback case studies using Live z-score training and a normative database. Journal of Neurotherapy, 14(1), 22-46. doi: 10.1080/10874200903543963 
Electro-Cap Internacional (1982). ECl electro-cap [apparatus]. https://electro-cap.com/ index.cfm/caps/

Fenger, T. N. (1998). Visual-motor integration and its relation to EEG neurofeedback brain wave patterns, reading, spelling, and arithmetic achievement in attention deficit disordered and learning disabled students. Journal of Neurotherapy, 3(1), 9-18. doi: 10.1300/J184v03n01_02

Fisher, W., Piazza, C. C., Bowman, L. G., Hagopian, L. P., Owens, J. C., \& Slevin, I. (1992). A comparison of two approaches for identifying reinforcers for persons with severe and profound disabilities. Journal of Applied Behavior Analysis, 25(2), 491-498. doi: 10.1901/jaba.1992.25-491

Fletcher, J. M., \& Grigorenko, E. L. (2017). Neuropsychology of learning disabilities: The past and the future. Journal of the International Neuropsychological Society, 23(9-10), 930940. doi: $10.1017 /$ S1355617717001084

Groeneveld, K. M., Mennenga, A. M., Heidelberg, R. C., Martin, R. E., Tittle, R. K., Meeuwsen, K. D., Walker, L. A., \& White, E. K. (2019). Z-score neurofeedback and heart rate variability training for adults and children with symptoms of attentiondeficit/hyperactivity disorder: A retrospective study. Applied Psychophysiology and Biofeedback. doi: 10.1007/s10484-019-09439-x

Hanslmayr, S., Sauseng, P., Doppelmayr, M., Schabus, M., \& Klimesch, W. (2005). Increasing Individual upper alpha power by neurofeedback improves cognitive performance in human subjects. Applied Psychophysiology and Biofeedback, 30(1), 110. doi: 10.1007/s10484-005-2169-8

Hughes, J. R., \& John, E. R. (1999). Conventional and quantitative electroencephalography in psychiatry. The Journal of Neuropsychiatry and Clinical Neurosciences, 11(2), 190208. doi: 10.1176/jnp.11.2.190

Jacobs, E. H. (2006). Neurofeedback treatment of two children with learning, attention, mood, social, and developmental deficits. Journal of Neurotherapy, 9(4), 55-70. doi: 10.1300/J184v09n04_06

Kaiser, D. A., \& Othmer, S. (2000). Effect of neurofeedback on variables of attention in a large multi-center trial. Journal of Neurotherapy, 4(1), 5-15. doi: 10.1300/J184v04n01_02

Kaufman, A. S., Flanagan, D. P., Alfonso, V. C., \& Mascolo, J. T. (2006). Test review: Wechsler intelligence scale for children, $4^{\text {th }}$ ed. (WISC-IV). Journal of Psychoeducational Assessment, 24(3), 278-295. doi: 10.1177/0734282906288389

Koberda, J. L., Moses, A., Koberda, L., \& Koberda, P. (2012). cognitive enhancement using 19-electrode $Z$-score neurofeedback. Journal of Neurotherapy, 16(3), 224-230. doi: 10.1080/10874208.2012.705769

Krigbaum, G., \& Wigton, N. L. (2014). When discussing neurofeedback, does modality matter? Neuroregulation, 1(1), 48-60. doi: 10.15540/nr.1.1.48

Krigbaum, G., \& Wigton, N. L. (2015a). A methodology of analysis for monitoring treatment progression with 19-channel z-score neurofeedback (19ZNF) in a single-subject design. Applied Psychophysiology and Biofeedback, 40(3), 139-149. doi: 10.1007/s10484015-9274-0

Krigbaum, G., \& Wigton, N. L. (2015b). A methodology of analysis for monitoring treatment progression with 19-channel z-score neurofeedback (19ZNF) in a single-subject design. Applied Psychophysiology and Biofeedback, 40(3), 139-149. doi: 10.1007/s10484015-9274-0

Kropotov, J. (2009). Methods of neurotherapy. In Quantitative EEG, event-related potentials and neurotherapy (pp. 469-505). San Diego, CA: Elsevier. doi: 10.1016/B978-0-12374512-5.00023-2 
Kropotov, J. (2016). Functional neuromarkers for psychiatry. Cambridge, MA: Academic Press Inc.

Lindsay, G. (2007). Educational psychology and the effectiveness of inclusive education/mainstreaming. British Journal of Educational Psychology, 77(1), 1-24. doi: 10.1348/000709906X156881

Mangum, A., Fredrick, L., Pabico, R., \& Roane, H. (2012). The role of context in the evaluation of reinforcer efficacy: Implications for the preference assessment outcomes. Research in Autism Spectrum Disorders, 6(1), 158-167. doi: 10.1016/j.rasd.2011.04.001

Medici, D. (2018). Neurofeedback versus pharmacological intervention in the treatment of childhood attention deficit/hyperactivity Disorder (ADHD): First spanish clinical neuropsychological study. American Journal of Applied Psychology, 7(5), 57. doi: 10.11648/j.ajap.20180705.11

Morris, S. B., \& DeShon, R. P. (2002). Combining effect size estimates in meta-analysis with repeated measures and independent-groups designs. Psychological Methods, 7(1), 105-125. doi: 10.1037/1082-989X.7.1.105

Ochi, Y., Laksanasopin, T., Kaewkamnerdpong, B., \& Thanasuan, K. (2017). Neurofeedback game for attention training in adults. 2017 10th Biomedical Engineering International Conference (BMEiCON), 1-5. doi: 10.1109/BMEiCON.2017.8229113

Orlando, P. C., \& Rivera, R. O. (2004). Neurofeedback for elementary students with identified learning problems. Journal of Neurotherapy, 8(2), 5-19. doi: 10.1300/J184v08n02_02

Orozco Cazco, G. H., Tejedor Tejedor, F. J., \& Calvo Álvarez, M. I. (2016). Meta-análisis sobre el efecto del software educativo en alumnos con necesidades educativas especiales [Meta-analysis on the effect of educational software on students with special educational needs]. Revista de Investigación Educativa, 35(1), 35. doi: 10.6018/rie.35.1.240351

Pérez-Elvira, R., Carrobles, J., López Bote, D., \& Oltra-Cucarella, J. (2019). Efficacy of Live zscore neurofeedback training for chronic insomnia: A single-case study. Neuroregulation, 6(2), 93-101. doi: 10.15540/nr.6.2.93

Pérez-Elvira, R., López Bote, D. J., Guarino, S., Agudo Juan, M., De León, R. J., Feiner, T., \& Perez, B. (2018). Neurometric results of a case series using live z-scores neurofeedback. International Journal of Psychophysiology, 131, S139-S140. doi: 10.1016/j.ijpsycho.2018.07.375

Piazza, C. C., Fisher, W. W., Hagopian, L. P., Bowman, L. G., \& Toole, L. (1996). Using a choice assessment to predict reinforcer effectiveness. Journal of Applied Behavior Analysis, 29(1), 1-9. doi: 10.1901/jaba.1996.29-1

Rastegar, N., Dolatshahi, B., \& Rezaee Dogahe, E. (2016). The effect of neurofeedback training on increasing sustained attention in veterans with posttraumatic stress disorder. Practice in Clinical Psychology, 4(2). doi: 10.15412/J.JPCP.06040204

Soutar, R. G. (2018). Holistic neurointegration: The new mind model. A bio-psycho-social qEEG guided neurofeedback method. Atlanta, GA: New Mind Academy.

Stoller, L. (2011). Z-score training, combinatorics, and phase transitions. Journal of Neurotherapy, 15(1), 35-53. doi: 10.1080/10874208.2010.545758

Thatcher, R. W. (2013). Latest developments in Live z-score training: Symptom check list, phase reset, and Loreta z-score biofeedback. Journal of Neurotherapy, 17(1), 69-87. doi: 10.1080/10874208.2013.759032

Walker, J. E. (2010). Recent advances in quantitative EEG as an aid to diagnosis and as a guide to neurofeedback training for cortical hypofunctions, hyperfunctions, disconnections, and hyperconnections: Improving efficacy in complicated neurological 
and psychological disorders. Applied Psychophysiology and Biofeedback, 35(1), 25-27. doi: 10.1007/s10484-009-9107-0

Walker, J. E. (2011). QEEG-guided neurofeedback for recurrent migraine headaches. Clinical EEG and Neuroscience, 42(1), 59-61. doi: 10.1177/155005941104200112

Wang, J.-R., \& Hsieh, S. (2013). Neurofeedback training improves attention and working memory performance. Clinical Neurophysiology, 124(12), 2406-2420. doi: 10.1016/j.clinph.2013.05.020

Wigton, N., \& Krigbaum, G. (2015). A review of qEEG-guided neurofeedback. Neuroregulation, 2(3), 149-155. doi: 10.15540/nr.2.3.149

Wigton, N. L. (2013). Clinical perspectives of 19-channel z-score neurofeedback: Benefits and limitations. Journal of Neurotherapy, 17(4), 259-264. doi: $10.1080 / 10874208.2013 .847142$

Wigton, N. L., \& Krigbaum, G. (2015). Attention, executive function, behavior, and electrocortical function, significantly improved with 19-channel z-score neurofeedback in a clinical setting: A pilot study. Journal of Attention Disorders, 23(4), 398-408. doi: $10.1177 / 1087054715577135$

Zoefel, B., Huster, R. J., \& Herrmann, C. S. (2011). Neurofeedback training of the upper alpha frequency band in EEG improves cognitive performance. Neuroimage, 54(2), 1427-1431. doi: 10.1016/j.neuroimage.2010.08.078

RECEIVED: April 12, 2020

ACCEPTED: August 25, 2020 


\section{Appendix}

CEC-Learning Items (Soutar, 2018)

\begin{tabular}{|c|l|}
\hline Item & \multicolumn{1}{|c|}{ Content } \\
\hline 11 & Poor short term memory \\
\hline 14 & List learning problems \\
\hline 29 & Can't recall more than one request \\
\hline 30 & Poor maths skills \\
\hline 31 & Poor reading comprenhesion \\
\hline 42 & Dyslexia \\
\hline 43 & Reads poorly \\
\hline 44 & Poor handwriting \\
\hline 48 & Difficult with task sequence \\
\hline 49 & Difficulty learning new words \\
\hline
\end{tabular}

Note: Reproduced with Richard Soutar's permission. 